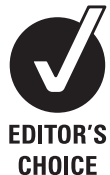

CHOICE

\title{
What explains the American disadvantage in health compared with the English? The case of diabetes
}

\author{
James Banks, ${ }^{1,2}$ Meena Kumari, ${ }^{3}$ James P Smith, ${ }^{4}$ Paola Zaninotto ${ }^{3}$
}

\begin{abstract}
- An additional appendix table $A$ is published online only. To view this file please visit the journal online (http://jech.bmj. com/content/66/3.toc)

${ }^{1}$ Department of Economics, University College London, London, UK

${ }^{2}$ Institute for Fiscal Studies, London, UK

${ }^{3}$ Department of Epidemiology, University College London, London, UK

${ }^{4}$ The RAND Corporation, Santa Monica, California, USA
\end{abstract}

\section{Correspondence to}

Dr James P Smith, the RAND Corporation, 1776 Main Street, Santa Monica, CA 90407, USA; smith@rand.org

Accepted 11 August 2010 Published Online First

1 October 2010

\section{ABSTRACT}

Background Middle-aged and older American men and women have almost twice the rate of diabetes of men and women in England. This differential was not explained by conventional risk factors including age, smoking, social position and body mass index (BMI).

Methods This study used large and representative samples of non-minority adults aged $52-85$ years taken from the 1999-2006 American National Health and Nutrition Examination Survey (NHANES) and the 2004 English Longitudinal Study of Aging. The surveys contain self-reported and objective biological disease markers of diabetes as well as indicators of major risk factors for diabetes including anthropometric measures of BMI, height and waist circumference.

Results The older American population has much higher rates of diabetes than the English population-a differential not yet explained, but this population also has higher waist circumference at each level of BMI than does the equivalent group in England. By controlling for such waist circumference differences and allowing for different effects of waist on diabetes in each country, approximately three-quarters of the country differences for women and 38\% among men can be explained.

Conclusions Higher rates of diabetes in the US old-age population than in England were largely accounted for by raised waist circumference and not BMI differences, especially among women. In addition, elevated diabetes risk associated with higher waist circumference in the USA as opposed to England could arise as a result of a number of different mechanisms. Investigation of the relative importance of such mechanisms is an important topic for further study.

Diabetes prevalence is approximately twice as high in the USA compared with England for both sexes. ${ }^{1}$ This difference was not due to differential disease self-reporting by country because the differential exists using either self-reports of doctor diagnosis or objective markers of diabetes. Conventional models of diabetes prevalence that included standard risk factors (smoking, age, social position) indicated that little of this international difference was explained by country differences in these risks. ${ }^{1}$ These differences remained substantial when body mass index (BMI) indicators of overweight or obese were included in the analyses, indicating that other factors were responsible. ${ }^{1}$

Recent data suggested that other aspects of body shape including waist circumference were different across countries. ${ }^{2}$ Increasing waist circumference was associated with the increasing risk of subsequent diabetes and was more predictive than BMI. ${ }^{3}$ The association of increased diabetes or its risk factors with shorter height has been described in many populations. ${ }^{5} 6$

Earlier analyses examining international differences in diabetes did not examine the role of waist circumference. ${ }^{1}$ We study its importance in the English Longitudinal Study of Ageing (ELSA) and the American National Health and Nutrition Examination Survey (NHANES), which are nationally representative cohorts with both BMI and waist circumference measurements. Our aim was to expand these models to consider the simultaneous risks of both BMI and waist circumference, and to assess how much of the difference in diabetes prevalence between England and America was explained by these body shape risks.

\section{METHODS}

\section{Data}

We used data from eight waves of NHANES, fielded between 1999 and 2006, and the 2004 second wave of ELSA. We combined NHANES waves from before and after ELSA 2004 to have comparable sample sizes. There was no statistically significant trend in diabetes prevalence over NHANES years 1999-2006 so NHANES was time comparable with ELSA. NHANES included data obtained through personal interviews, physical and laboratory examinations for people 2 months and older. For age comparability, we restricted our NHANES sample to respondents between 52 and 85 years old-age 52 years was the minimum age in ELSA and age 85 years was the maximum age in NHANES. To ensure that country differences were not due to high rates of diabetes among AfricanAmericans or Hispanic individuals in America or Asian and black immigrants in England, our analysis was restricted to non-Hispanic white individuals. There were 4570 non-Hispanic white NHANES respondents in this age range.

ELSA contained respondents recruited from three separate years of the Health Survey for England providing representative English samples aged 50 years and over in 2002. Health data were supplemented by the collection of social and economic data. ${ }^{8}$ ELSA protocols included interview visits followed by nurse visits when saliva and blood samples were drawn. Blood samples were analysed for glycosylated haemoglobin, also available in NHANES. There were 6888 respondents in our ELSA analytical sample.

NHANES is a nationally representative survey of Americans less than 85 years of age with a high response rate. The baseline response rate in 2005-6 was $80 \%$ and $77 \%$ for those receiving medical examinations. Its sample characteristics closely match the current population surveys. 
NHANES is a repeated cross-sectional survey so it was unaffected by sample attrition (see http://www.cdc.gov/nchs/ nhanes/response rates CPS .htm).

We examined impacts of differential response bias and sample attrition in ELSA on estimates of disease prevalence including diabetes by comparing estimates with those from the Health Survey for England. ${ }^{9}$ Differences in diabetes prevalence between the two countries were equally large in cross-sectional national surveys indicating that disease prevalence rates were not significantly biased due to differential attrition or response rates.

\section{Measures of diabetes prevalence}

Both surveys collected data on individual diabetes self-reports of the form 'Did a doctor ever tell you that you had diabetes...' as well as glycosylated haemoglobin (HbA1c), a measure of the percentage of haemoglobin molecules bound to glucose. Although not usually a screener for diabetes, HbA1c is highly correlated with fasting plasma glucose levels. ${ }^{9}{ }^{10}$ While there is no strict diagnosis threshold value, we took values greater than or equal to $6.5 \%$ as indicating clinical diabetes (an international threshold recently approved). ${ }^{11}$ Our results were insensitive to the specific thresholds chosen. Respondents were classified as having diabetes if either they answered affirmatively to the selfreported diabetes question or their $\mathrm{HbA1c}$ levels were $6.5 \%$ or greater. Our measures were thus unaffected by country differences in undiagnosed diabetes.

\section{Measures of BMI and waist risk}

Height and weight measurements were taken during nurseconducted physical examinations so objective BMI measures were computed free of errors in self-reports. ${ }^{12}$ We experimented with two BMI measures-categorical indicators of whether respondents were obese (BMI $\geq 30$ ) or overweight (BMI $\geq 25$ and $<30)$ and continuous BMI measures $\left(\mathrm{kg} / \mathrm{m}^{2}\right)$ entered as quadratics. As height may have an independent effect, ${ }^{13}$ height in metres was entered as a quadratic.

BMI provides a crude index of adiposity by not considering central fat mass, which is more strongly associated with disease risk. Our data contained both BMI and central fat mass-objectively measured waist circumference in centimetres. ${ }^{14}$ Similar to BMI, we experimented with two measures of waist risk. The first categorised respondents into three waist risk groups (low, medium and high) in which centimetre cut-off points differed by gender. ${ }^{15} 16$ The male groups were low $(<94 \mathrm{~cm})$, moderate $(94-101 \mathrm{~cm})$ and high risk $(\geq 102 \mathrm{~cm})$, whereas for women cut-off points were low $(<80 \mathrm{~cm})$, moderate $(80-88 \mathrm{~cm})$ and high risk $(\geq 88 \mathrm{~cm})$. Our second measure was waist in centimetres, entered as a quadratic.

\section{Other risk factors}

American education was separated into high school or less (0-12 years), more than high school but not a college graduate (13-15 years) and college or more (16 years or greater). The English three-way education division was qualified to a level lower than ' $O$ ' level or equivalent (typically 0-11 years), qualified to a level lower than ' $A$ ' level or equivalent (typically $12-13$ years of schooling), and a higher qualification (more than 13 years). Our surveys collected several health-related behaviours including smoking status (ever smoked) and marital status (single or married).

\section{Statistical methods}

We estimated six models of diabetes prevalence separately for men and women. We began with a baseline model pooled across countries with no covariates except an indicator of country-our 'unadjusted' model or country differences in diabetes prevalence we tried to explain. The subsequent five models labelled 1 to 5 all included quadratics in age and in height, and indicator variables described above for education, marital status and smoking.

We were particularly interested in the role of BMI and waist circumference combined with how they were measured. Models 1 to 5 differed in how we specified them. Model 1 used conventional BMI categorical indicators of obesity and overweight; model 2 had the same BMI categorical indicators and added waist categorical indicators of moderate and high waist risk; model 3 had continuous BMI entered as a quadratic; model 4 had continuous BMI and continuous waist both as quadratics; model 5 had the same model as model 4 but in addition allowed the effects of continuous BMI and continuous waist to differ between America and England.

We relied on ordinary least squares regression models of prevalence, estimated using Stata statistical software. As our models contained interaction terms, logistic regressions do not provide a straightforward interpretation. ${ }^{17}$ Nevertheless our conclusions from the non-interactive models were unchanged if multiple logistic or multivariate probit models were used.

\section{RESULTS}

Table 1 lists means and standard deviations of variables and displays results of statistical tests for country differences. American male prevalence was $16 \%$ compared with $11 \%$ for English men and 14\% for American women compared with 7\% for English women. Both country differences in diabetes prevalence were statistically significant at the $1 \%$ level.

Conventional diabetes risk factors were similar in the American and English populations examined. On average Americans are only a little taller, have slightly higher BMI and are approximately 3 years older. The English were less educated and more likely to have smoked, risk factors that normally increase diabetes. ${ }^{3} 1819$ The only attribute offering some explanatory promise was waist circumference. American men have almost $3 \mathrm{~cm}$ larger waists than English men, and the waists of American women are $5 \mathrm{~cm}$ bigger than English women $(p<0.01)$.

Figures $1-3$ present age standardised distribution of waist risk (low, moderate and high) among those whose BMI is below 25, between 25 and 30 and 30 and over separately by gender in both countries. Age standardisation eliminated impacts of country differences in age distributions. Using age groups 50-59, 60-69, $70-79,80$ years and over, age-weighted BMI and waist risks were calculated. To capture risk variation, individuals were placed into three BMI groups and three waist risk groups defined above. Supplementary appendix table A, available online only, includes statistical tests of differences in waist and BMI between countries.

American men were more obese than English men (31.6\% compared with $26.7 \%$ ) but less overweight (44.0\% compared with $48.3 \%$ ) with no difference in normal weight. Female obesity differences were even smaller (32.9\% of Americans compared with $30.6 \%$ among English women), a disparity not statistically significant and not large enough to explain a two-toone diabetes prevalence differential between countries. Compared with English women, American women were less overweight $(32.1 \%$ compared with $38.7 \%$ ) and more of normal weight (35.0\% compared with $30.8 \%$ ). Given these BMI distributions, it is unsurprising that BMI alone cannot explain higher diabetes rates among Americans.

Marginal distributions of waist risk within each BMI group were more to the disadvantage of American men than BMI 
Table 1 Means and standard deviations

\begin{tabular}{lcccr}
\hline & $\begin{array}{l}\text { English men } \\
\text { Mean (SD) }\end{array}$ & $\begin{array}{l}\text { English women } \\
\text { Mean (SD) }\end{array}$ & $\begin{array}{l}\text { American men } \\
\text { Mean (SD) }\end{array}$ & $\begin{array}{c}\text { American women } \\
\text { Mean (SD) }\end{array}$ \\
\hline Diabetes prevalence & $0.11(0.32)$ & $0.07(0.25)$ & $0.16^{* *}(0.36)$ & $0.14^{* *}(0.34)$ \\
BMl & $27.84(4.14)$ & $28.04(5.29)$ & $28.12^{*}(5.00)$ & $28.01(6.17)$ \\
Waist (cm) & $101.59(11.49)$ & $90.99(12.59)$ & $104.44^{* *}(13.17)$ & $95.94^{* *}(14.40)$ \\
Height (m) & $1.73(0.07)$ & $1.59(0.07)$ & $1.74(0.07)$ & $1.60(0.07)$ \\
Age (years) & $65.54(8.68)$ & $65.81(8.98)$ & $68.87^{* *}(10.68)$ & $68.97^{* *}(10.44)$ \\
Single & $0.20(0.40)$ & $0.36(0.48)$ & $0.24(0.43)$ & $0.48^{* *}(0.50)$ \\
Ever smoked & $0.72(0.45)$ & $0.56(0.50)$ & $0.68(0.47)$ & $0.44^{* *}(0.50)$ \\
Medium education & $0.27(0.44)$ & $0.33(0.47)$ & $0.25(0.43)$ & $0.32(0.47)$ \\
Low education & $0.42(0.49)$ & $0.42(0.49)$ & $0.23^{* *}(0.42)$ & $0.32^{* *}(0.47)$ \\
\hline
\end{tabular}

Non-Hispanic white individuals aged 52-85 years from the 2004 ELSA and 1999-2006 NHANES samples, respectively

*Statistically significant at $0.05 ;{ }^{* *}$ statistically significant at 0.01 difference between American and English.

differences and female disparities are striking- $56.0 \%$ of English women have high waist risk, whereas the comparable fraction among Americans was $69.4 \%$ - a statistically significant difference. These differences are in accord with recent evidence from smaller more specialist studies in which body shape was measured using three-dimensional body scanning equipment in both countries.

If there was a one-to-one correspondence between BMI and waist risk categories, there would be little reason to prefer one. But this is not true. Among the overweight in figure 2, 56\% of American men have high waist risk compared with $41 \%$ of English men, while $87 \%$ of American women have high waist risk compared with $61 \%$ of English women. Even among those with normal weight, the fraction with raised waist risk was not trivial for US women-40.6\% of Americans who were neither overweight nor obese were categorised as high waist risk compared with $8.9 \%$ among equivalent English women. The only BMI category with little additional information obtained

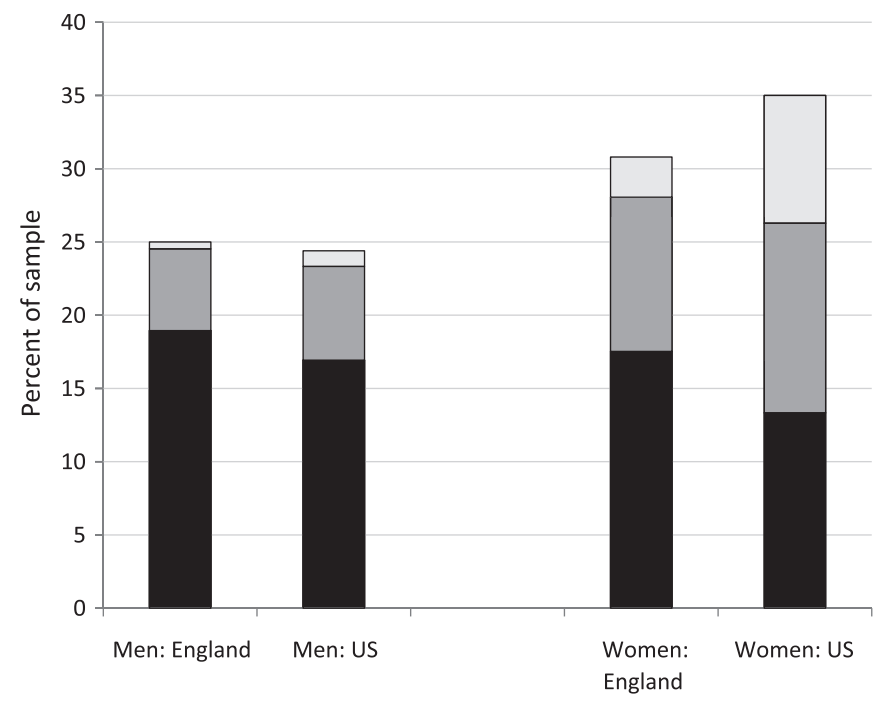

$\square$ High waist risk $\square$ Moderate waist risk $\square$ Low waist risk

Notes:

1. Non-hispanic whites aged 52-85 from the 2004 ELSA and 1999-2006 NHANES samples respectively

2. Low waist risk defined as $<94 \mathrm{~cm}(\mathrm{M})<80 \mathrm{~cm}(\mathrm{~W})$; Moderate waist risk defined as $94-102 \mathrm{~cm}(\mathrm{M}), 80-88 \mathrm{~cm}(\mathrm{~W})$; High waist risk defined as $\geq 102 \mathrm{~cm}(\mathrm{M}), \geq 88 \mathrm{~cm}(\mathrm{~W})$

3. Statistics are age-standardised and weighted

4. The height of each bar in these figures tells us the fraction of each demographic group who are of normal, over-weight, and obese BMI respectively. The relative size of the shaded areas within each bar informs us about those who are of low, moderate, and high waist risk within that BMl category.

Figure 1 Distribution of waist risk for those with BMI below 25. from waist were the obese (figure 3). At least $95 \%$ of respondents labelled obese were simultaneously labelled high waist risk.

Figure 4 summarises the ability of factors to explain country differences in diabetes prevalence using our six models. For each model, the solid bar indicates our estimate of 'unexplained' prevalence rate differences between America and England, while the lines represent $95 \% \mathrm{CI}$ around that estimate. The unadjusted specification reported a baseline model pooled across countries with just the country dummy (=1 indicates US). This model highlighted unadjusted country differences in diabetes-4.8\% for men and $7.3 \%$ for women.

Subsequent models in the next five rows in figure 4 sequentially expand the set of covariates while maintaining the assumption that the effects of covariates are the same in both countries. All models below unadjusted included quadratic controls for age and height, indicator variables for education groups, single and smoking. Models 1-4 varied the way in which BMI and waist circumference were included in models.

Model 1 estimates in figure 4 represented the traditional approach to measuring BMI-two indicators for whether one was overweight or obese. This model did not explain any between-country prevalence difference-some factors (age, $\mathrm{BMI}$ ) predict higher US diabetes prevalence, other factors (education, height) predict the opposite with combined effects largely offsetting. Model 2 highlights the impacts of adding categorical variables to capture waist risk-these effects are

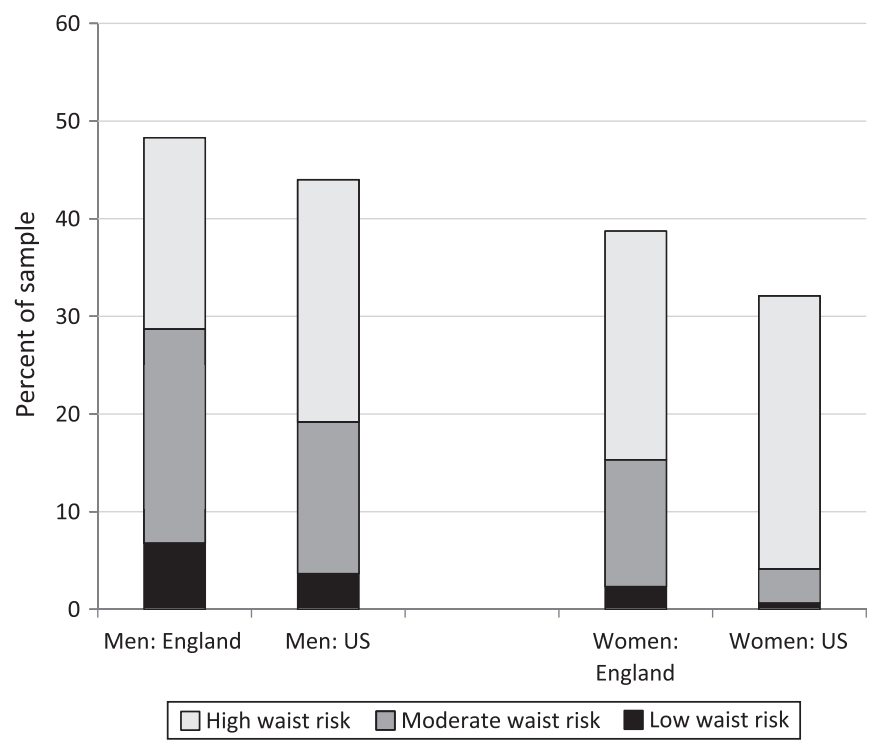

Figure 2 Distribution of waist risk for those with BMI between 25 and 30. 


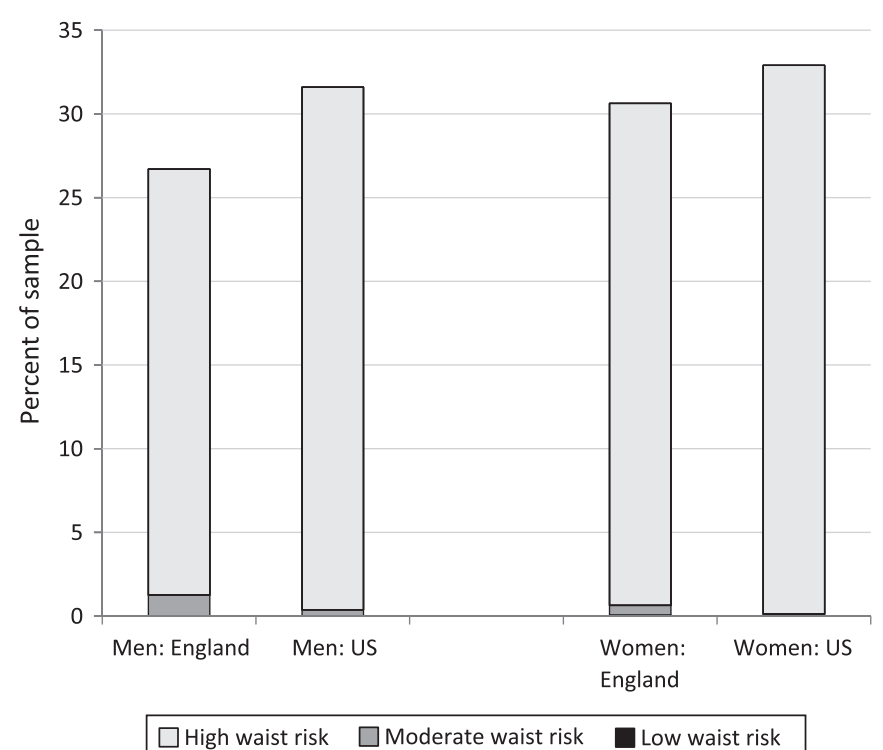

Figure 3 Distribution of waist risk for those with BMI 30 or over.

quite modest in reducing unexplained country prevalence differences.

Model 3 was similar to model 1 except continuous quadratic BMI replaced standard obese and overweight categories controls. Estimated country prevalence differences were unchanged. Model 4 shows effects of including continuous quadratic measures of BMI and waist risk. With both shape variables measured continuously instead of as categories, we explained one quarter of the original male difference in prevalence and approximately $60 \%$ of the female difference. Continuous BMI by itself does not help explain country differences (another illustration of BMI deficiency), but a combination of continuous measures of BMI and waist significantly reduced country differences in 'unexplained' diabetes prevalence.

Models 1-4 did not allow effects of BMI or waist on predicting diabetes to vary by country. Our preferred model 5

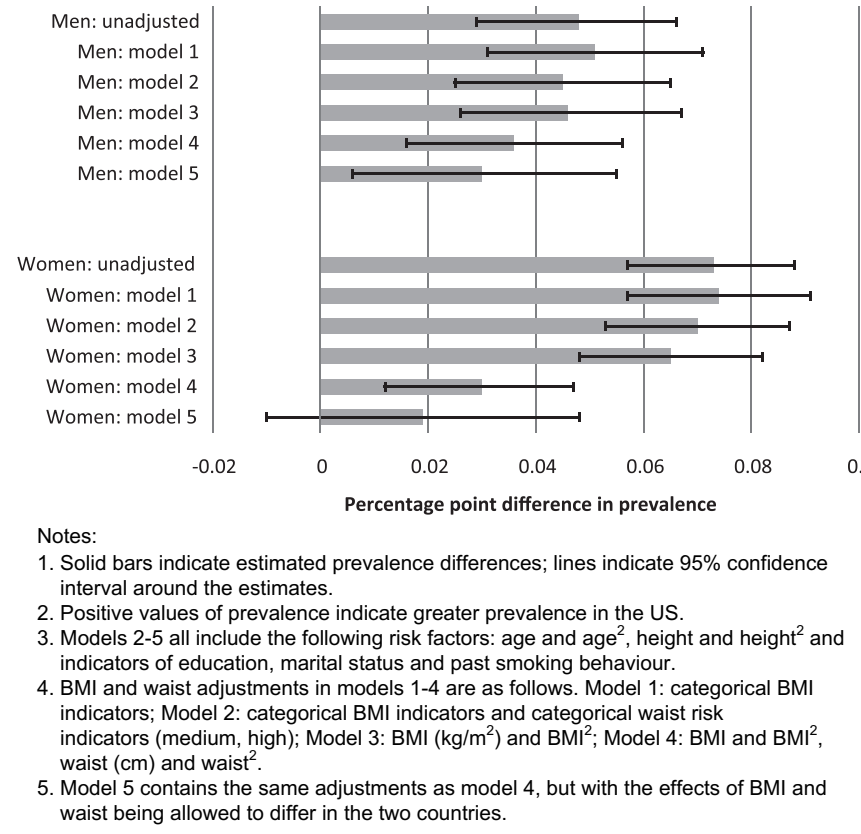

Figure 4 Estimated diabetes prevalence difference between US and England. allowed estimated impacts of both body shape measures to vary between countries. The remaining unexplained male country difference was 3 percentage points and 1.9 percentage points for women. As indicated by $95 \%$ CI in figure 4 , female country difference in diabetes was no longer statistically significant. Control variables in this model explained $38 \%$ of country difference in male prevalence and $74 \%$ of female differences.

The regression models underlying model 5 in figure 4 are presented in table 2. Estimates for non-anthropometric variables were as expected. ${ }^{20}$ The probability of diabetes rose with age at a decreasing rate, increased height is associated with lower diabetes risk, and smoking and marital status did not matter. ${ }^{21} 22$

After controlling for waist, we found no statistically significant effect of BMI for American or English men or women. In contrast to weak BMI effects, waist risk was strongly associated with diabetes, with risk increasing in waist circumference. Based on a likelihood ratio test, quadratic waist terms were statistically significant for American men and women implying that the consequences of raised waist are higher for them compared with the English.

\section{DISCUSSION}

We found significant roles for height and waist on diabetes prevalence. The mechanisms by which height reduces diabetes risk are unclear. Adult stature reflects childhood growth patterns and an association of short stature with type 2 diabetes indicates that impaired childhood growth leads to adult insulin resistance and diabetes. ${ }^{23}$ Mechanisms include poor nutrition in childhood. ${ }^{24}$ There may be reverse causation at older ages, in which co-morbidity with diabetes is associated with height reduction, but we found no significant age interactions indicating that these influences are unlikely to vary by country. While height was related to diabetes risk, height cannot explain country differences because average height was approximately the same in both countries.

In contrast, waist circumference explained a substantial proportion of higher diabetes in America for men and virtually all differences for women, but continuous measures were required to capture differences in diabetes risks between the two populations.

Table 2 Models of diabetes prevalence

\begin{tabular}{lrr}
\hline & \multicolumn{1}{c}{$\begin{array}{c}\text { B (SE) } \\
\text { Men }\end{array}$} & \multicolumn{1}{c}{$\begin{array}{l}\text { B (SE) } \\
\text { Women }\end{array}$} \\
\hline USA & $0.0302^{*}(0.013)$ & $0.0191(0.015)$ \\
Age & $0.0114^{* *}(0.002)$ & $0.0049^{* *}(0.002)$ \\
Age squared & $-0.0003^{* *}(0.000)$ & $-0.0000^{*}(0.000)$ \\
Height & $-0.3168^{* *}(0.079)$ & $-0.2958^{* *}(0.065)$ \\
Height squared & $-0.0040(0.604)$ & $-0.2081(0.578)$ \\
Single & $0.0160(0.015)$ & $0.0045(0.008)$ \\
Ever smoke & $0.0050(0.010)$ & $-0.0073(0.008)$ \\
Med education & $-0.0041(0.012)$ & $0.0090(0.009)$ \\
Low education & $-0.0150(0.011)$ & $0.0267^{* *}(0.009)$ \\
BMI & $0.0016(0.004)$ & $-0.0028(0.003)$ \\
BMI squared & $-0.0005(0.000)$ & $-0.0003(0.000)$ \\
USA BMI & $-0.0014(0.006)$ & $-0.0033(0.004)$ \\
USA BMI squared & $0.0009^{*}(0.000)$ & $0.0000(0.000)$ \\
Waist & $0.0037^{*}(0.002)$ & $0.0023(0.001)$ \\
Waist squared & $0.0001^{*}(0.000)$ & $0.0002^{* *}(0.000)$ \\
USA waist & $0.0013^{*}(0.002)$ & $0.0024^{*}(0.002)$ \\
USA waist squared & $-0.0001(0.000)$ & $-0.0000(0.000)$ \\
Constant & $-0.0424^{*}(0.020)$ & $-0.0414^{* *}(0.016)$ \\
\hline
\end{tabular}

*Statistically significant at $0.05 ;{ }^{* *}$ statistically significant at 0.01 . 
Evidence cites increasing prevalence of obesity with increased burden of disease, ${ }^{22}$ but little was explained here by obesity categorised by BMI index. ${ }^{1}$ BMI provides a crude index of adiposity by not considering central fat mass, which is more strongly associated with disease risk. Acknowledging this is important when making health comparisons across different populations. Our data suggested that in each BMI category a greater proportion of Americans were at increased disease risk and that waist circumference played the key role.

There are several potential mechanisms, including different rates of physical activity through exercise or activities of daily life, diet differences, or a more adverse psychosocial environment in America, all associated with central adiposity and type 2 diabetes. ${ }^{182526}$ Adverse stressful environments may be associated with the development of central obesity. ${ }^{27}$

Evidence between central fat accumulation and diabetes risk is striking. ${ }^{3}$ Compared with waist hip ratio, waist circumference is a better marker of visceral fat. ${ }^{28}$ Fat cells located in the viscera display distinct cellular features compared with fat cells elsewhere, and a specific dysfunction of these cells may be the pathophysiological basis for negative consequences of abdominal obesity. Central fat cells have a higher turnover rate of triglycerides, produce more pro-inflammatory and metabolic markers than fat cells from other depots. They are involved in the mobilisation of free fatty acids into portal circulation, impaired liver function leading to insulin resistance and diabetes.

Our results were the same if we limited analyses to those whose diabetes onset occurred after age 34 years so our conclusions are not driven by country differences in type 1 diabetes. Our results thus apply most directly to type 2 diabetes.

ELSA and NHANES allowed international comparisons in large representative cohorts, a key strength of this paper, but some limitations exist. Several covariates that might influence diabetes risk were unavailable-equivalent collection of physical activity or dietary information and medication intakes. Evidence suggests that while more recently developed antihypertensive medications are not diabetogenic, older treatments increase diabetes risk. ${ }^{29}$ We cannot examine antihypertensive medication in detail, but speculate that, while physical activity and dietary intake may play a role, antihypertensive medication prescribing

\section{What is already known on this subject}

- Middle-aged and older Americans have twice the rate of diabetes prevalence compared with England.

- This differential is not explained by conventional risk factors including age, smoking, social position and BMI.

\section{What this study adds}

- Middle-aged and older American men and particularly American women have much higher waist circumference compared with the English.

- High waist circumference measured continuously was far more important than BMI in predicting diabetes prevalence.

- Higher diabetes in America than in England was largely accounted for by higher American waist circumference and larger impacts of high waist on diabetes in America. practices are similar in America and England and are unlikely to affect these international differences.

Two powerful conclusions follow from this research. Higher American rates of diabetes compared with England were largely due to a combination of high waist among Americans and a higher impact of waist risk among Americans. The reasons may be related to measures that were not available such as objective measures of physical activity and dietary intake.

This combination of higher waist and higher risk of high waist in America explained virtually all excess diabetes of American women and almost half for American men. BMI alone failed to explain any of these differences. Besides this key substantive conclusion, our results showed that modelling diabetes successfully requires good measures of waist risk, particularly in populations that might differ in distributions of body fat like the US and English. Similar issues should be investigated for other cardiovascular disease and countries.

Funding Support was received from a grant from the NIA to RAND and from the NIA and the Economic and Social Research Council to the Institute for Fiscal Studies.

Competing interests None declared

Provenance and peer review Not commissioned; externally peer reviewed.

\section{REFERENCES}

1. Banks J, Marmot M, Oldfield Z, et al. Disease and disadvantage in the United States and in England. JAMA 2006;295:2037-45.

2. Wells JCK, Cole TJ, Bruner D, et al. Body shape in American and British adults: between country and inter-ethnic comparisons. Int J Obesity 2008;32:152-9.

3. Perry IJ, Wannamethee SG, Walker MK, et al. Prospective study of risk factors for development of non-insulin dependent diabetes in middle aged British men. BMJ 1995;310:560-4

4. Schulze MB, Heidemann C, Schienkiewitz A, et al. Comparison of anthropometric characteristics in predicting the incidence of type 2 diabetes in the EPIC-Potsdam study. Diabetes Care 2006;29:1921-3.

5. Brown DC, Byrne CD, Clark PMS, et al. Height and glucose tolerance in adult subjects. Diabetologia 1991;34:531-3.

6. Kumari M, Chandola T, Brunner E. A nonlinear relationship of generalized and central obesity with diurnal cortisol secretion in the Whitehall II Study. J Clin Endocrinol Metab 2010;95(9):4415-23

7. Centers for Disease Control and Prevention, National Center for Health Statistics. National Health and Nutrition Examination Survey data sets and related documentation. http://www.cdc.gov/nchs/about/major/nhanes/datalink.htm (accessed 9 Jan 2006).

8. Banks J, Muriel A, Smith JP. Disease prevalence, disease incidence, and mortality in the United States and in England. Demography 2010;47:0ctober.

9. Davidson M, Schriger D, Peters A, et al. Relationship between fasting plasma glucose and glycosylated hemoglobin. Potential for false positive diagnoses of type 2 diabetes using new diagnostic criteria. JAMA 1999;281:1203-10.

10. Goldman N, Lin I, Weinstein $\mathrm{M}$, et al. Evaluating the quality of self-reports of hypertension and diabetes. J Clin Epidemiol 2003;56:148-54.

11. International Expert Committee. The International Expert Committee report on the role of the $\mathrm{A} 1 \mathrm{C}$ assay in the diagnosis of diabetes. Diabetes Care 2009;32:1327-34.

12. Gunnell D, Berney L, Holland P, et al. How accurately are height, weight and leg length reported by the elderly, and how closely are they related to measurements recorded in childhood? Int J Epidemiol 2000;29:456-64.

13. Han TS, Feskens EJ, Lean ME, et al. Associations of body composition with type 2 diabetes mellitus. Diabet Med 1998;15:129-35.

14. Turcato E, Bosello O, Di Francesco V, et al. Waist circumference and abdominal sagittal diameter as surrogates of body fat distribution in the elderly: their relation with cardiovascular risk factors. Int J Obes Relat Metab Disord 2000;24:1005-10.

15. Lean ME, Han TS, Morrison CE. Waist circumference as a measure for indicating need for weight management. BMJ 1995;311:158-61.

16. Flegal KM. Waist circumference of healthy men and women in the United States Int $J$ Obes 2007:31:1134-9.

17. Knol JK, van der Tweel J, Grobbee DE, et al. Estimating interaction effects on an additive scale between continuous determinants in a logistic regression model. Int $J$ Epidemiol 2007;36:1111-18.

18. Holbrook TL, Barrett-Connor E, Wingard DL. A prospective population-based study of alcohol use and non-insulin-dependent diabetes mellitus. Am J Epidemiol 1990:132:902-9.

19. Willi C, Bodenmann P, Ghali WA, et al. Active smoking and the risk to type 2 diabetes: a systematic review and meta-analysis. JAMA 2007;298:2654-64.

20. Smith JP. Nature and causes of male diabetes trends, undiagnosed diabetes, and the SES health gradient. Proc Natl Acad Sci U S A 2007;104:13225-31. 
21. Cowie CC, Eberhardt MS. Sociodemographic characteristics of persons with diabetes. In: National Diabetes Data Group, eds. Diabetes in America. 2nd edn. Bethesda, MD: National Diabetes Data Group, National Institutes of Health, National Institute of Diabetes and Digestive and Kidney Diseases, 1995:95-1468.

22. Kumari M, Head J, Marmot M. Prospective study of social and other risk factors for incidence of type 2 diabetes in the Whitehall II study. Arch Intern Med 2004;164:1873-80.

23. Eriksson JG, Forsen T, Tuomilehto J, et al. Early adiposity rebound in childhood and risk of type 2 diabetes in adult life. Diabetologia 2003;46:190-4.

24. Wadsworth $\mathbf{M}$, Hardy R, Paul A, et al. Leg and trunk length at 43 years in relation to childhood health, diet and family circumstances: evidence from the 1946 national birth cohort. Int J Epidemiol 2002;31:383-90.
25. Folsam AR, Kushi LH, Hong CP. Physical activity and incident diabetes mellitus in postmenopausal women. Am J Public Health 2000;90:134-8.

26. Brunner EJ, Mosdøl A, Witte DR, et al. Dietary patterns and 15-y risks of major coronary events, diabetes, and mortality. Am J Clin Nutr 2008:87:1414-21.

27. Epel ES, McEwen B, Seeman T, et al. Stress and body shape: stress-induced cortisol secretion is consistently greater among women with central fat. Psychosom Med 2000:62:623-32.

28. Han TS, McNeill G, Seidell JC, et al. Predicting intra-abdominal fatness from anthropometric measures: the influence of stature. Int J Obes Relat Metab Disord 1997;21:587-93

29. Mancia G, Grassi G, Zanchetti A. New-onset diabetes and antihypertensive drugs J Hypertens 2006;24:3-10.

\section{DIFFERENTIAL DIAGNOSIS}

\section{Trustworthy guidance on your iPhone}

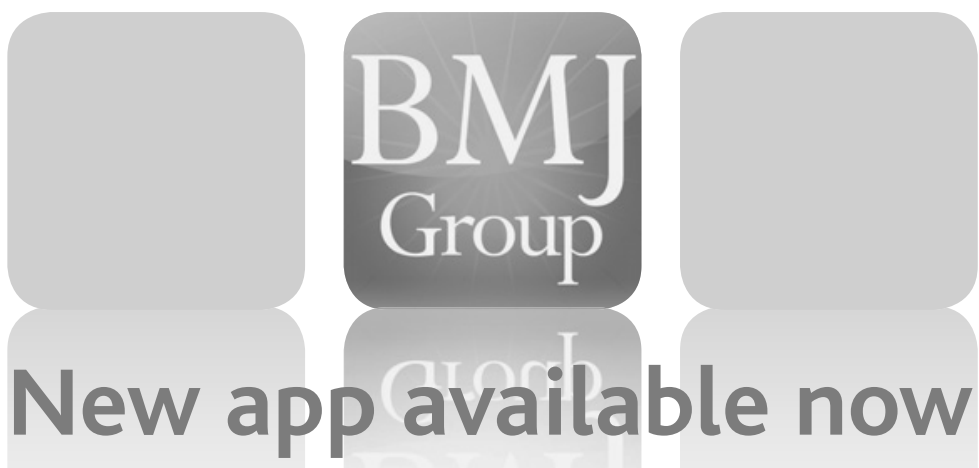

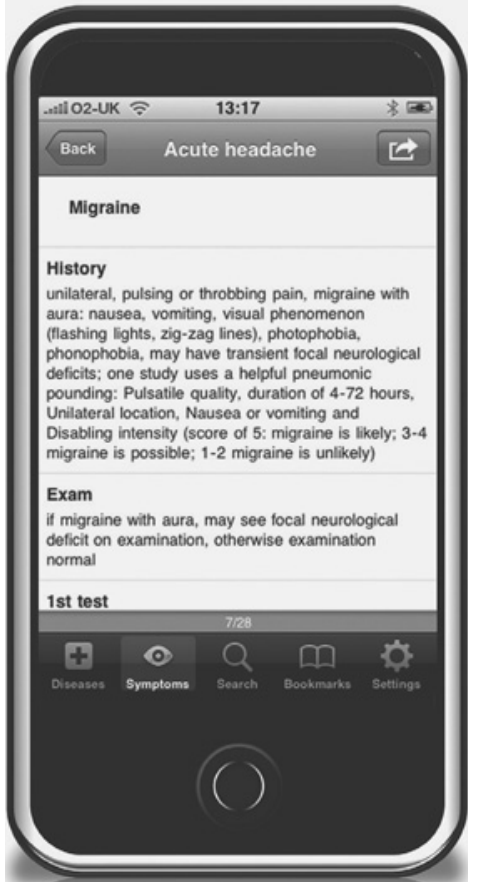

\section{Find out more at bestpractice.bmj.com/differentials}

